\title{
The effect of hydroxy safflower yellow A on coronary heart disease through Bcl-2/Bax and PPAR- $\gamma$
}

\author{
DAYAN ZHOU, ZONGJIE QU, HAO WANG, YONG SU, YAZHU WANG, \\ WEIWEI ZHANG, ZHE WANG and QIANG XU
}

Department of Cardiology, The Fifth People's Hospital of Chongqing, Chongqing 400062, P.R. China

Received December 20, 2015; Accepted February 2, 2017

DOI: $10.3892 /$ etm.2017.5414

\begin{abstract}
The aim of the present study was to investigate the effect of hydroxy safflower yellow A (HSYA) on coronary heart disease through assessing the expression of B-cell lymphoma 2 $(\mathrm{Bcl}-2) / \mathrm{Bcl}-2$-like protein 4 (Bax) and peroxisome proliferator-activated receptor (PPAR)- $\gamma$. Coronary heart disease was induced in male Bama miniature swines via thoracoscope to serve as an animal model. Coronary heart disease swine were lavaged with 20 or $40 \mathrm{mg} / \mathrm{kg}$ HSYA. The mRNA levels of tumor necrosis factor (TNF)- $\alpha$, interleukin (IL)-1 $\beta$, IL-6, IL-10, cyclooxygenase-2 (COX-2) and inducible nitric oxide synthase (iNOS) were detected using reverse transcription-quantitative polymerase chain reaction. The protein expression of Bcl-2, Bax, PPAR- $\gamma$, phosphorylation of Janus kinase (JAK) 2 and phosphorylation of signal transducer and activator of transcription (STAT) 3 were detected using western blot analysis. Treatment with HSYA significantly suppressed the mRNA levels of IL-1 $\beta$ $(\mathrm{P}<0.01)$, IL-6 $(\mathrm{P}<0.01)$, TNF- $\alpha(\mathrm{P}<0.01), \mathrm{COX}-2(\mathrm{P}<0.01)$ and iNOS $(\mathrm{P}<0.01)$, and significantly increased IL-10 mRNA level in the coronary heart disease model $(\mathrm{P}<0.01)$. Furthermore, HSYA treatment significantly decreased the $\mathrm{Bcl}-2 / \mathrm{Bax}$ ratio $(\mathrm{P}<0.01)$ in the coronary heart disease model group, and enhanced the phosphorylation of JAK2/STAT3 pathway $(\mathrm{P}<0.01)$. However, HSYA had no significant effect on the expression of PPAR- $\gamma$ protein. The results of the present study suggest that HSYA is able to weaken coronary heart disease via inflammation, Bcl-2/Bax and the PPAR- $\gamma$ signaling pathway.
\end{abstract}

\section{Introduction}

Coronary heart disease is a dynamic process in which there are interactions between endothelial dysfunction and

Correspondence to: Mr. Qiang Xu, Department of Cardiology, The Fifth People's Hospital of Chongqing, 24 Renji Road, Nanan, Chongqing 400062, P.R. China

E-mail: qiangxucq@yeah.net

Key words: hydroxy safflower yellow A, coronary heart disease, inflammation, B-cell lymphoma 2, B-cell lymphoma-like protein 4, peroxisome proliferator-activated receptor- $\gamma$ inflammatory responses (1). Vascular endothelial cells (VECs), which are pinacocytes located on the surface of blood vessel lumen, are able to precept hemodynamic and hematogenous signal changes, and synthesize and secrete various vaso-active substances (2). VECs maintain the stability of the internal environment of blood vessels by releasing vessel dilators and constriction factors (3). If there is an imbalance in these factors, vasculature may form atheromatous plaques under the actions of inflammatory factors and cardiovascular risk factors (4). Inflammatory mediators are the factors that initiate the occurrence and progression of coronary heart disease (5).

Janus kinase signal transducer and activator of transcriptions (JAK/STAT) is a member of the intracellular transduction signal pathway family (6). As an important signal pathway in this family, JAK2/STAT3 serves an essential role in coronary heart disease (7).

As a key factor in signal transduction process, peroxisome proliferator-activated receptor (PPAR)- $\gamma$ regulates the expression of transcription proteins during glycometabolism and lipogenesis (8). PPAR- $\gamma$ is able to regulate lipid metabolism, adipocyte differentiation and insulin sensitivity (9). A previous study demonstrated that PPAR- $\gamma$ is an important cytokine that participates in glycometabolism and lipid metabolism. It was also found that PPAR- $\gamma$ is able to improve lipid levels and regulate xanthoma cell formations (10), and therefore may be able to ameliorate atherosclerosis. It has also been demonstrated that PPAR- $\gamma$ is able to inhibit atherosclerosis (10). PPAR- $\gamma$ is expressed in all vascular cells and serves an essential role in cell proliferation, migration, differentiation, the activation of macrophages and endothelial inflammation (11).

Recent pharmacodynamic studies have demonstrated that hydroxy safflower yellow A (HSYA) exhibits anti-platelet aggregation, anti-thrombogenesis, lipid-decreasing, anti-atherosclerosis, anti-infarction, inhibiting myocardial apoptosis and anti-oxidative stress functions, and is able to increase coronary blood-flow volumes, improve micro-circulation and hemorheology, and promote resistance to anoxia $(12,13)$. HSYA also has protective actions against acute ischemic myocardium $(12,13)$. The underlying mechanisms of HSYA are associated with the regulation of nitric oxide (NO), 6-ketone-prostaglandin F1a,thromboxane B2 and angiotensin II to increase blood-supply and oxygen supply of myocardium and reduce myocardial cell injury and apoptosis (13). The aim of the present study was to investigate the effect of HSYA on 
coronary heart disease through assessing the levels of B-cell lymphoma 2 (Bcl-2)/Bcl-2-like protein 4 (Bax) and PPAR- $\gamma$.

\section{Materials and methods}

Animals and coronary artery stenosis model. The present study was approved by the Animal Welfare and Ethics Committee of Fifth People's Hospital of Chongqing (Chongqing, China). Male Bama miniature swine (age, $10 \pm 2$ months; weight, $23 \pm 2 \mathrm{~kg}, \mathrm{n}=30$ ) were obtained from the Animal Center of the Chongqing Medical University (Chongqing, China) and housed in a temperature controlled room $\left(22-24^{\circ} \mathrm{C}\right), 55-60 \%$ humidity with a 12-h dark/cycle and free access to food and water.

Swine were anesthetized with intramuscular ketamine (35 mg/kg; Sigma-Aldrich; Merck KGaA, Darmstadt, Germany) and diazepam (1 mg/kg, Sigma-Aldrich; Merck $\mathrm{KGaA}$ ) prior to surgery. A 2-cm main operation incision was made at the fourth rib line in the left axillary level, and a thoracoscope was inserted at the left sternal 2 level. A 1-cm auxiliary operation incision was made at the left side of the clavicle midline between the fourth rib line. Two vessel forceps were subsequently inserted into each hole to push the lung lobes. Pericardium was separated and cut $2 \mathrm{~cm}$ to acquire the heart. The forward diagonal and ventricular branches of the left anterior descending (LAD) artery were acquired. The LAD artery was gently separated into the proximal diagonal branch and the left main branch, $1 \mathrm{~cm}$ apart. The LAD artery was placed on the operating table at $37^{\circ} \mathrm{C}$ and the blood vessels were ligated with silk suture. In sham group, swine were only anesthetized with intramuscular ketamine $(35 \mathrm{mg} / \mathrm{kg}$; Sigma-Aldrich; Merck KGaA, Darmstadt, Germany) and diazepam (1 mg/kg, Sigma-Aldrich; Merck KGaA) prior to surgery.

Experimental animals. A total of 30 Bama miniature swine were randomly divided into four groups as follows: Sham group (sham; $n=6$ ), coronary heart disease model group (model; $n=8$ ), low dose HSYA group (HSYA-L; $n=8$ ) and high dose HSYA group (HSYA-H; $n=8$ ). Swine in the sham and model groups were lavaged with normal saline. Swine in the HSYA-L and HSYA-H groups were lavaged with 20 or $40 \mathrm{mg} / \mathrm{kg}$ of HSYA, respectively, for $6 \mathrm{~h}$. HSYA was purchased from Shanxi Huahui Kai Tak Pharmaceutical Co., Ltd. (Shanxi, China) and its chemical structure is shown in Fig. 1.

Left ventricular ejection fraction (LVEF), left ventricular systolic internal diameter (LVIDs), blood urea nitrogen (BUN) plasma creatinine (Scr) and urine levels. Ultrasound (iE33 xMatrix; Philips Healthcare, Andover, MA, USA) was performed to survey and calculate LVEF and LVIDs level. The levels of BUN (C013-2), Scr (C011-2) and urine (C035-2) were measured using ELISA kits (Nanjing Jiancheng Chemical Industrial Co., Ltd., Nanjing, China).

Reverse transcription-quantitative polymerase chain reaction $(R T-q P C R)$. After treatment with HSYA, swines were anesthetized with intramuscular ketamine $(35 \mathrm{mg} / \mathrm{kg}$; Sigma-Aldrich; Merck KGaA) and diazepam (1 mg/kg; Sigma-Aldrich; Merck $\mathrm{KGaA}$ ), and sacrificed by intravenous injection with $10 \% \mathrm{KCl}$ immediately followed blood emptying. Frozen heart tissue

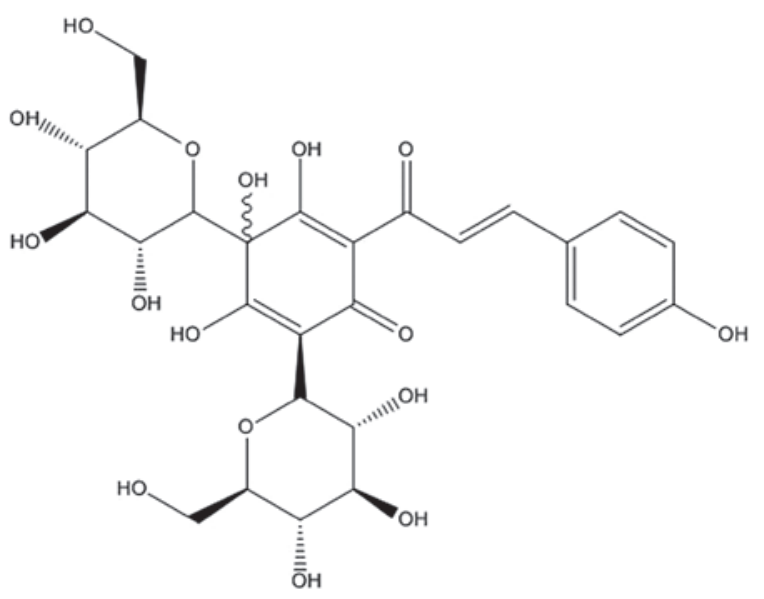

Figure 1. Chemical structure of hydroxy safflower yellow A.

samples was acquired and washed with PBS. Total RNA was extracted from the frozen heart tissue samples using TRIzol (Thermo Fisher Scientific, Inc., Waltham, MA, USA) according to the manufacturer's protocol. cDNA synthesis was performed using $2 \mu \mathrm{g}$ total RNA and Moloney-murine leukemia virus reverse transcriptase (Invitrogen; Thermo Fisher Scientific, Inc.). A total of $1 \mu \mathrm{l}$ cDNA was used for PCR with SYBR Premix ExTaq (Invitrogen; Thermo Fisher Scientific, Inc.) on an iCycler iQ Real-time Detection System (Applied Biosystems; Thermo Fisher Scientific, Inc.). The primer sequences for cycloxygenase-2 (COX-2) and inducible nitric oxide synthase (iNOS) are listed in Table I. PCR amplification was performed as follows: Initial denaturation at $95^{\circ} \mathrm{C}$ for $3 \mathrm{~min}$, followed by 40 cycles with denaturation at $95^{\circ} \mathrm{C}$ for $15 \mathrm{sec}$ and annealing at $60^{\circ} \mathrm{C}$ for $30 \mathrm{sec}$. $\beta$-actin was used as a reference gene. Analysis of relative gene expression data using the $2^{-\Delta \Delta \mathrm{Cq}} \operatorname{method}(14)$.

ELISA. After treatment with HSYA, peripheral blood was collected and serum was collected after centrifugation at $2,000 \mathrm{xg}$ for $10 \mathrm{~min}$ at $4^{\circ} \mathrm{C}$. Serum was used to measure IL-1 $\beta$ (H002), IL-6 (H007), IL-10 (H009) and TNF- $\alpha$ (H052) levels using ELISA Kits (Nanjing institute of biological engineering, Nanjing, China) according to the manufacturer's instructions.

Western blotting. Heart tissue samples were lysed in ice-cold lysis buffer (radioimmunoprecipitation assay buffer; Beyotime Institute of Biotechnology) and protease inhibitor mixture (phenylmethanesulfonyl fluoride; Beyotime Institute of Biotechnology) for $30 \mathrm{~min}$. The resulting supernatant was collected and boiled following 15 min centrifugation at $12,000 \mathrm{xg}$ at $4^{\circ} \mathrm{C}$. Protein concentrations of the extracts were measured by bicinchoninic acid assay (Nanjing Jiancheng Chemical Industrial Co., Ltd.). A total of $50 \mu \mathrm{g}$ whole protein was separated by $8-12 \%$ SDS-PAGE and subsequently transferred onto nitrocellulose membranes. Membranes were blocked with 5\% non-fat milk in TBS and Tween-20 (TBST) for $1 \mathrm{~h}$ at $37^{\circ} \mathrm{C}$, and incubated with the following antibodies: Anti-Bax (sc-6236, 1:3,000; Santa Cruz Biotechnology, Inc., Dallas, TX, USA), anti-Bcl-2 (sc-783, 1:2,000; Santa Cruz Biotechnology, Inc.), anti-phosphorylated (p)-JAK2 (3776, 1:4,000; Cell Signaling Technology, Inc., Danvers, 
Table I. Primers used for reverse transcription-quantitative polymerase chain reaction.

\begin{tabular}{lcc}
\hline Primer & Forward sequence & \multicolumn{1}{c}{ Reverse sequence } \\
\hline COX-2 & 5'-TCGCTGTGCCTGATGATTCC-3' & 5'-CTTATGATCTCGTTTCCGTC-3' \\
iNOS & 5'-CCAAGCCCTCACCTACTTCC-3' & 5'-CTCTGAGGGCTGACACAAGG-3' \\
$\beta$-actin & 5'-CACGAAACTACCTTCAACTCC-3' & 5'-CATACTCCTGCTTGCTGATC-3'
\end{tabular}

COX, cyclooxygenase; iNOS, inducible nitric oxide synthase.
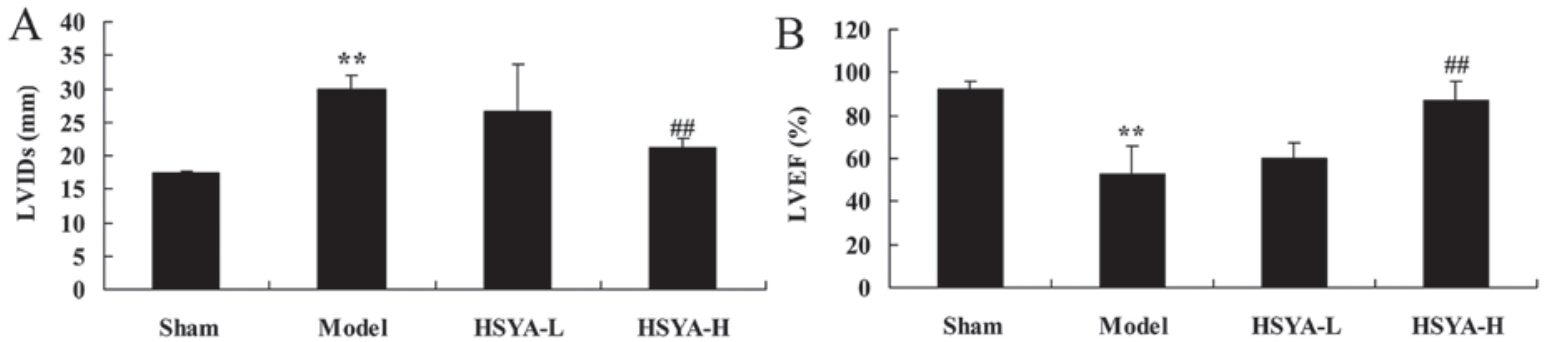

Figure 2. Effect of HSYA on LVIDs and LVEF in coronary heart disease model. The effect of HSYA on (A) LVIDs and (B) LVEF in a coronary heart disease model. ${ }^{* *} \mathrm{P}<0.01$ vs. sham; ${ }^{\# /} \mathrm{P}<0.01$ vs. model. HSYA, hydroxy safflower yellow A; LVEF, left ventricular ejection fraction; LVID, left ventricular systolic internal diameter; sham, sham group; model, coronary heart disease model group; HSYA-L, low dose HSYA group; HSYA-H, high dose HSYA group.

MA, USA), anti-p-STAT3 (9145, 1:2,000; Cell Signaling Technology, Inc.), anti-PPAR- $\gamma$ (sc-9000, 1:500; Santa Cruz Biotechnology, Inc.) and $\beta$-actin (sc-7210, 1:1,000) at $4^{\circ} \mathrm{C}$ overnight, followed by washing with TBST three times for 10 min. Membranes were subsequently probed with the appropriate secondary antibodies (sc-2004; 1:5,000; Santa Cruz Biotechnology) at room temperature for $2 \mathrm{~h}$ and washed with TBST. Protein was and visualized by a BeyoECL Plus (Beyotime Institute of Biotechnology) and calculated by Image J Image analysis software 3.0 (National Institutes of Health, Bethesda, MD, USA).

Statistical analysis. Data were presented as the mean \pm standard error of the mean using SPSS 21.0 (IBM Corp., Armonk, NY, USA). Groups were evaluated using the Kruskal-Wallis test for nonparametric independent samples by one-way analysis of variance. $\mathrm{P}<0.05$ was considered to indicate a statistically significant difference.

\section{Results}

Effect of HSYA on LVIDs and LVEF in the coronary heart disease model. Compared with the swine in the sham group, the LVIDs level of the model group was significantly increased $(\mathrm{P}<0.01$; Fig. 2A). The LVEF were significantly lower in the model group compared with the shame group $(\mathrm{P}<0.01$; Fig. 2B). Treatment with a high dose of HSYA was able to significantly reverse this effect $(\mathrm{P}<0.01$; Fig $2 \mathrm{~A}$ and $\mathrm{B})$.

Effect of HSYA on biochemical composition in the coronary heart disease model. Following $6 \mathrm{~h}$ of treatment, a significant increase was observed in levels of $\operatorname{Scr}(\mathrm{P}<0.01$; Fig. 3A) BUN $(\mathrm{P}<0.01$; Fig. $3 \mathrm{~B})$, and urine concentration $(\mathrm{P}<0.01$; Fig. $3 \mathrm{C})$ in the model group compared with the sham group. Treatment with high-does HSYA, however, significantly ameliorated these effects (all $\mathrm{P}<0.01$; Fig. 3).

Effect of HSYA on the levels of interleukin $(I L-1 \beta, I L-6, I L-10$ and tumor necrosis factor (TNF)- $\alpha$ in the coronary heart disease model. mRNA Levels of IL-1 $\beta$, IL- 6 and TNF- $\alpha$ were significantly higher in the model group compared with the sham group (all $\mathrm{P}<0.01$; Fig. 4A-C), and IL-10 expression was significantly downregulated in the model group compared with the sham group $(\mathrm{P}<0.01$; Fig. 4D). Treatment with high-dose HSYA significantly reversed the increase in IL-1 $\beta$, IL- 6 and TNF- $\alpha$ levels, and the decrease in IL-10 level in the model group $(\mathrm{P}<0.01$; Fig. 4).

Effect of HSYA on the levels of COX-2 mRNA in the coronary heart disease model. The results of RT-qPCR revealed that the expression of COX-2 mRNA was significantly higher in the model group compared with the sham group $(\mathrm{P}<0.01$; Fig. 5). Treatment with high-dose HSYA, however, significantly suppressed the COX-2 mRNA levels in the model group $(\mathrm{P}<0.01$; Fig. 5).

Effect of HSYA on the levels of iNOS mRNA in the coronary heart disease model. A significant increase was observed in levels of iNOS mRNA in the model group compared with the sham group $(\mathrm{P}<0.01$; Fig. 6). The HSYA-H group exhibited a inhibited a significant downregulation in iNOS mRNA compared with the model group $(\mathrm{P}<0.01$; Fig. 6).

Effect of HSYA on the Bax/Bcl-2 ratio and PPAR $-\gamma$ protein expression in the coronary heart disease model. Results of western blot analysis revealed that the $\mathrm{Bax} / \mathrm{Bcl}-2$ ratio were significantly higher in the model group compared with the sham group ( $\mathrm{P}<0.01$; Fig. 7 ). The $\mathrm{Bax} / \mathrm{Bcl}-2$ ratio was significantly 

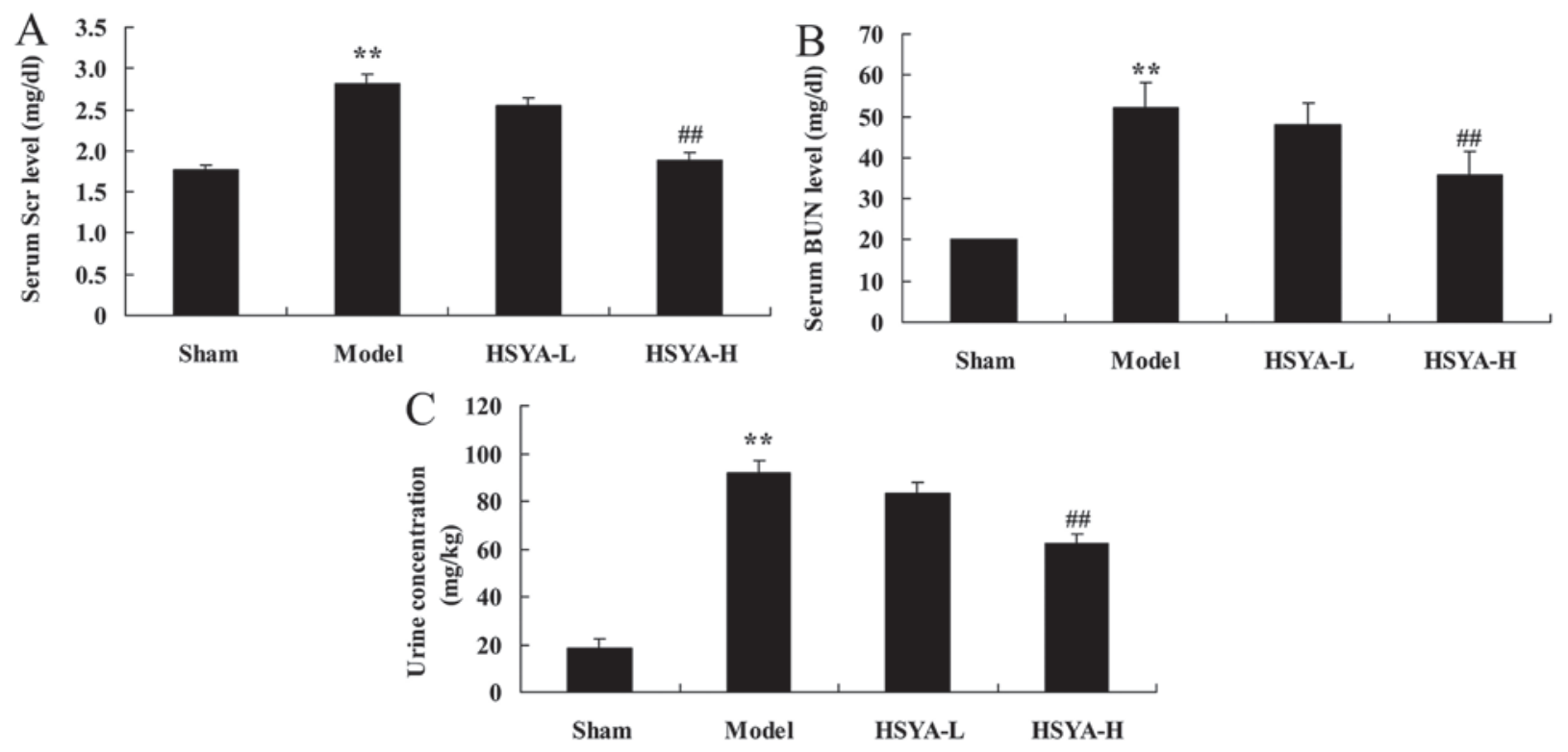

Figure 3. Effect of HSYA on biochemical composition in coronary heart disease model. The effect of HSYA on (A) Scr, (B) BUN and (C) urine in a coronary heart disease model. ${ }^{* *} \mathrm{P}<0.01$ vs. sham; ${ }^{\# \#} \mathrm{P}<0.01$ vs. model. HSYA, hydroxy safflower yellow A; Scr, plasma creatine; BUN, blood urea nitrogen; sham, sham group; model, coronary heart disease model group; HSYA-L, low dose HSYA group; HSYA-H, high dose HSYA group.
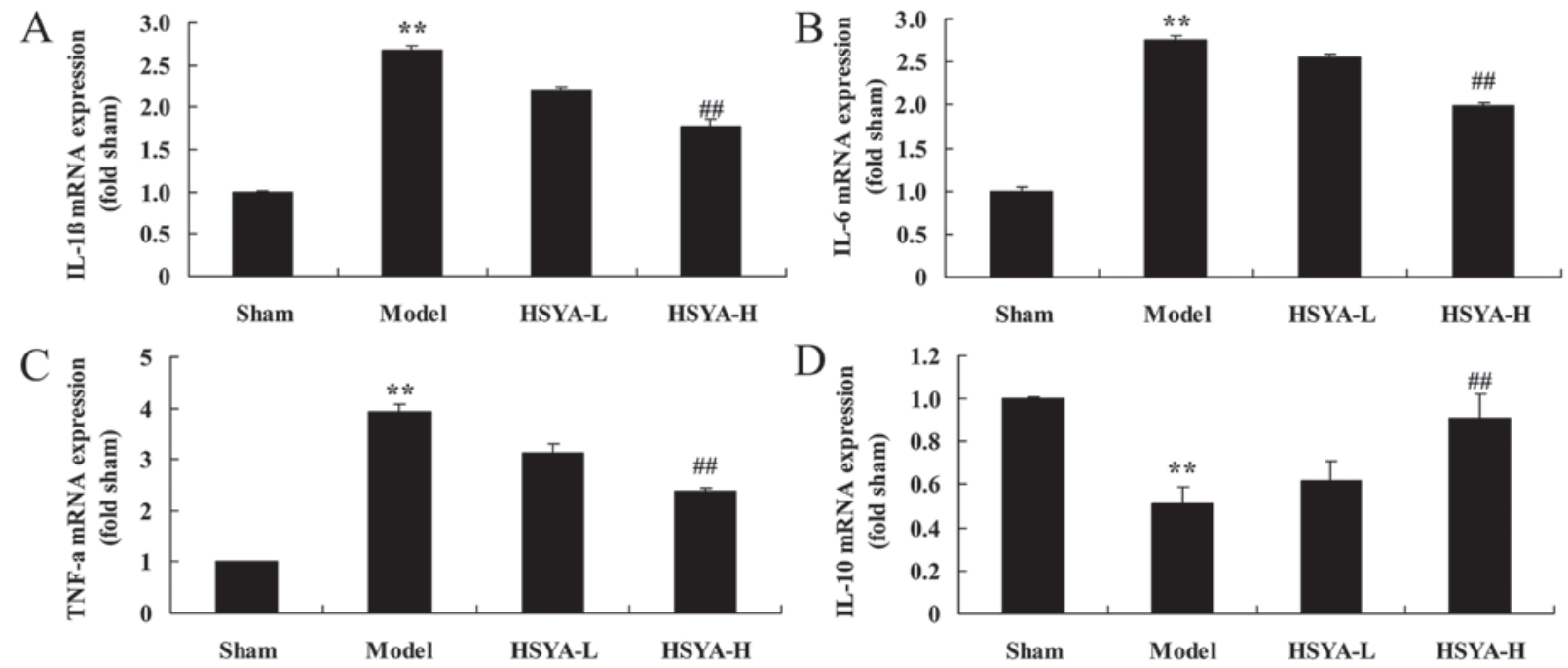

Figure 4. Effect of HSYA on the mRNA levels of IL-1 $\beta$, IL-6, IL-10 and TNF- $\alpha$ in coronary heart disease model. The effect of HSYA on the mRNA levels of (A) IL-1 $\beta$, (B) IL-6, (C) TNF- $\alpha$ and (D) IL-10 in a coronary heart disease model. ${ }^{* *} \mathrm{P}<0.01$ vs. sham; ${ }^{* \#} \mathrm{P}<0.01$ vs. model. HSYA, hydroxy safflower yellow A; IL, interleukin; TNF, tumor necrosis factor; sham, sham group; model, coronary heart disease model group; HSYA-L, low dose HSYA group; HSYA-H, high dose HSYA group.

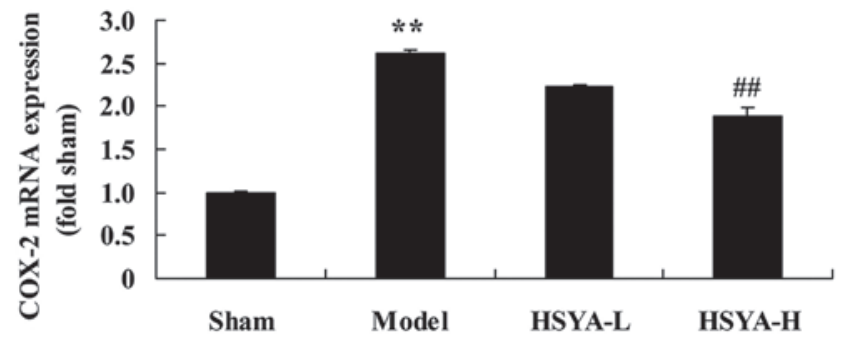

Figure 5. Effect of HSYA on the mRNA levels of COX-2 in a coronary heart disease model. ${ }^{* *} \mathrm{P}<0.01$ vs. sham; ${ }^{\# \#} \mathrm{P}<0.01$ vs. model. HSYA, hydroxy safflower yellow A; COX, cyclooxygenase; sham, sham group; Model, coronary heart disease model group; HSYA-L, low dose HSYA group; HSYA-H, high dose HSYA group.

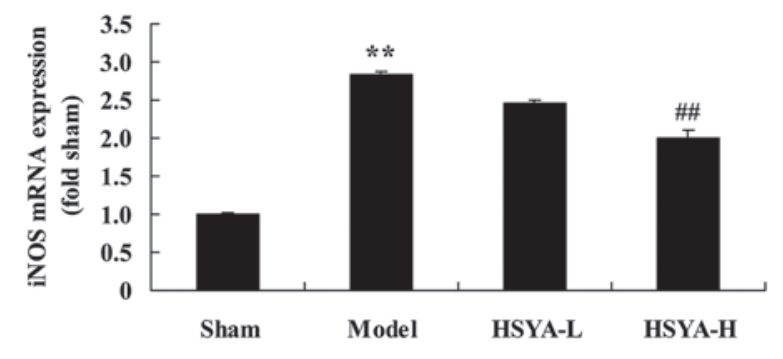

Figure 6. Effect of HSYA on the mRNA levels of iNOS in a coronary heart disease model. ${ }^{* *} \mathrm{P}<0.01$ vs. sham; ${ }^{\# \#} \mathrm{P}<0.01$ vs. model. HSYA, hydroxy safflower yellow A; iNOS, inducible nitric oxide synthase; sham, sham group; model, coronary heart disease model group; HSYA-L, low dose HSYA group; HSYA-H, high dose HSYA group. 

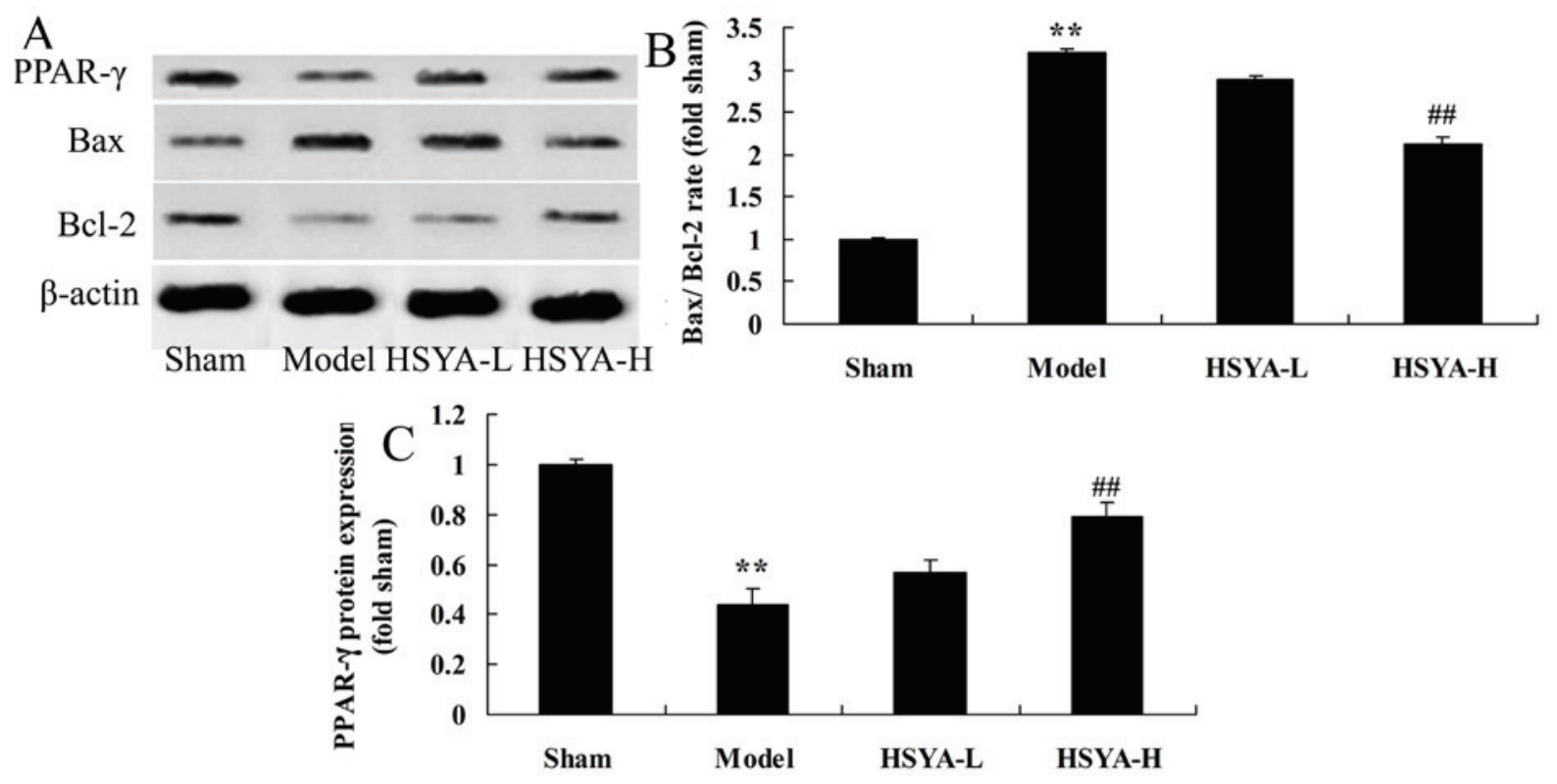

Figure 7. Effect of HSYA on the Bcl-2/Bax ratio and PPAR- $\gamma$ in coronary heart disease model. The effect of HSYA on the Bcl-2/Bax and PPAR- $\gamma$ protein expression by (A) western blotting assays and statistical analysis of (B) Bcl-2/Bax and (C) PPAR- $\gamma$ protein expression in coronary heart disease model. ${ }^{* *} \mathrm{P}<0.01$ vs. sham; \#\# P<0.01 vs. model. HSYA, hydroxy safflower yellow A; Bcl-2, B-cell lymphoma 2; Bax, Bcl-2-like protein 4; Sham, sham group; Model, coronary heart disease model group; HSYA-L, low dose HSYA group; HSYA-H, high dose HSYA group.
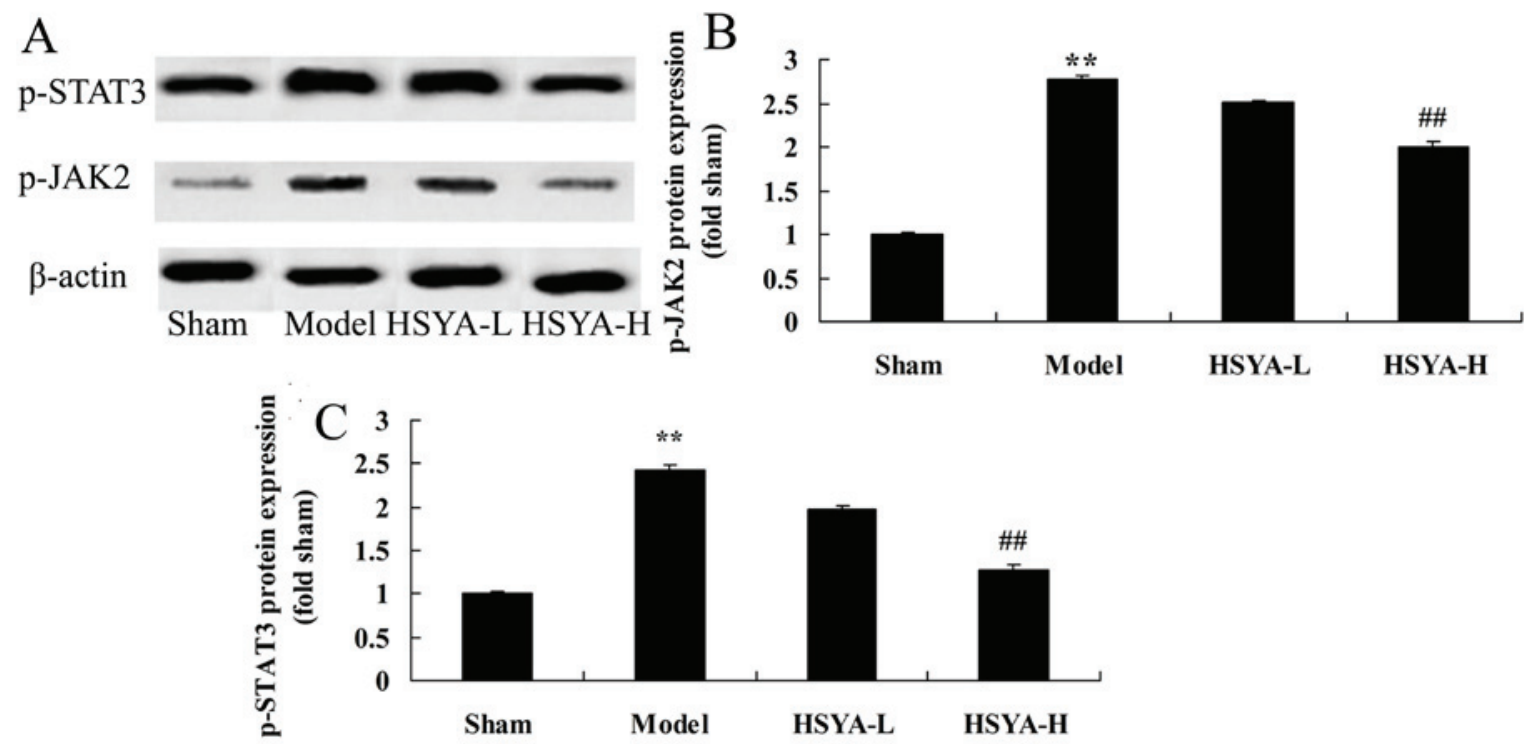

Figure 8. Effect of HSYA on the phosphorylation of JAK2 and STAT3 protein expression in coronary heart disease model. (A) Western blotting and statistical analysis of (B) p-JAK2 and (C) p-STAT3 protein expression in the coronary heart disease model ${ }^{* *} \mathrm{P}<0.01 \mathrm{vs}$. sham; ${ }^{\# \#} \mathrm{P}<0.01$ vs. model. HSYA, hydroxyl safflower yellow A; JAK, Janus kinase; STAT, signal transducer and activator of transcription; p-, phosphorylated; sham, sham group; model, coronary heart disease model group; HSYA-L, low dose HSYA group; HSYA-H, high dose HSYA group.

lower in the HSYA-H group compared with the model group $(\mathrm{P}<0.01$; Fig. 7). PPAR- $\gamma$ protein expression were significantly lower in the model group compared with the sham group $(\mathrm{P}<0.01$; Fig. 7). However, PPAR- $\gamma$ protein expression was significantly higher in the HSYA-H group compared with the model group $(\mathrm{P}<0.01$; Fig. 7$)$.

Effect of HSYA on the expression of p-JAK2 and p-STAT3 protein in the coronary heart disease model. The results of western blot analysis revealed that p-JAK2 and p-STAT3 levels were significantly higher in the model group compared with sham group $(\mathrm{P}<0.01 ;$ Fig. 8). However, this increase was ameliorated by treatment with high-dose HSYA ( $\mathrm{P}<0.01$; Fig. 8$)$.

\section{Discussion}

Previous research has demonstrated that the inflammatory response is associated with coronary heart disease (4). Inflammatory markers and endothelial function are both effective indicators for predicting and treating coronary heart 
disease (4). IL-6 and other pro-inflammatory cytokines are able to regulate the synthesis of coronary heart disease and IL-6 is fundamental to this process (15). Similar to CRP, the imbalance of IL-6 may influence vary diseases (5). In atherosclerosis, IL-6 primarily acts on blood vessel endothelium, causing it to be impaired and therefore activating a series of inflammatory responses (5), which leads to coronary atherosclerotic cardiomyopathy or the conversion of stable coronary heart disease to unstable (15). In the present study, HSYA was demonstrated to significantly ameliorate LVEF, LVIDs and biochemical composition, and suppressed the expression of IL-1 $\beta$, IL-6, IL-10 and TNF- $\alpha$ in a coronary heart disease model. Zhou et al (13) reported that HSYA weakens myocardial apoptosis following acute myocardial infarction in rats via affecting Bax/Bcl-2 and PPAR- $\gamma$. Zhang et al (12) demonstrated that HSYA significantly reduced the IL- $1 \beta$, IL- 4, TNF- $\alpha$, COX-2, iNOS, and increased IL-10 mRNA levels in Alzheimer's disease via phosphorylation of the JAK2/STAT3 pathway.

Endothelial dysfunction is the first stage of coronary heart disease and manifests as a decrease in endothelium-dependent vasodilation function, NO synthesis and the disorder of angiokinesis factors (16). NO is synthesized under the function of L-arginine. It is an important endothelium-derived relaxing factor, which serves an important role in regulating angiokinesis (17). Endothelium-dependent vasodilatation is damaged following coronary atherosclerosis, whereas contraction function is strengthened,resulting in the reduction of myocardial perfusion and myocardial ischemia (17). Furthermore, NO is able to protect blood vessels from injury and inhibit inflammatory responses and thrombogenesis (16). It is also able to inhibit leukocyte adhesion, platelet aggregation and enable vascular smooth muscles to remain in a quiescent state (16). In the presence of clinical risk factors such as hypertension, diabetes and hyperlipidemia, the epithelial defense functions in blood vessels are disordered (18). In the present study, HSYA significantly downregulated iNOS and COX-2 mRNA levels in a coronary heart disease model.

PPAR $\gamma$ is expressed in all vascular cells (mononuclear leucocytes, macrophages and endothelial cells) and vascular smooth muscle cells (10). It serves a fundamental role in cell proliferation, migration, differentiation, the activation of macrophages and vascular endothelium inflammation (10). PPAR $\gamma$ activated by vascular endothelial dysfunction may inhibit TNF- $\alpha$, IL-1 $\beta$ and IL- 6 secreted by monocyte-macrophages as well as the expression of platelet-activating factor receptor, VCAM-1, iNOS and scavenger receptor A (11). It is able to lower or limit the progress of chronic inflammation, maintain the stability of membrane permeability, inhibit the activity of smooth muscle cells and reduce inward flows of calcium (11). Therefore, vascular contractile responses and smooth muscle cell proliferation may be inhibited and elastic resistance may be reduced (9). The results of the present study suggest that HSYA significantly upregulated PPAR- $\gamma$ protein expression in swine with coronary heart disease. Zhou et al (13) reported that the effect of HSYA weakens myocardial apoptosis following acute myocardial infarction in rats through $\mathrm{Bax} / \mathrm{Bcl}-2$ and PPAR- $\gamma$.

Cell apoptosis is a type of programmed cell death regulated by genes, which is induced by initiation of the apoptosis signal pathway and the expression of related genes (19). The mitochondrial pathway is one of the most important apoptosis signal transduction pathways (20). The occurrence and progression of cell apoptosis is regulated by various genes, of which Bcl-2 serves a fundamental role via the mitochondrial pathway (19). Bcl-2 is an anti-apoptosis gene while Bax is the pro apoptotic gene (20). Additionally, HSYA significantly reduced the $\mathrm{Bax} / \mathrm{Bcl}-2$ ratio in swine with coronary heart disease. Zhou et al (13) reported that HSYA is able to weaken myocardial apoptosis following acute myocardial infarction in rats via the expression of Bax/Bcl-2 and PPAR- $\gamma$.

The JAK/STAT pathway is associated with the inflammatory response, oxidative stress, cellular damage and apoptosis (19). IL-6 is able to worsen myocardial hypertrophy via activating the JAK/STAT pathway (21). The JAK/STAT pathway mediates the reduction in synthesis of the surface-active materials of pulmonary epithelial cells caused by oxidative stress (22). Coronary heart disease is typically accompanied by mitochondrial damage, which worsens coronary heart disease (23). Oxidative damage to mitochondria includes changes in permeability, swelling, oxidative phosphorylation and the release of pro-apoptotic proteins (24). Recent studies revealed that the activation or inhibition of the JAK2/STAT3 pathway may have a significant effect on oxidative stress injuries and mitochondrial function $(23,25)$. In the present study, HSYA significantly suppressed p-JAK2 and p-STAT3 protein expression in swine with coronary heart disease. Zhang et al (12) demonstrated that HSYA was able to significantly reduce the expression of IL-1 $\beta$, IL-4, TNF- $\alpha$, COX-2, iNOS, and increase levels of IL-10 mRNA in patients with Alzheimer's disease via the phosphorylation of the JAK2/STAT3 pathway.

In conclusion, the neuroprotective effects of HSYA significantly improved LVEF and LVIDs and biochemical composition, and suppressed the levels of IL-1 $\beta$, IL-6, IL-10, TNF- $\alpha$, iNOS and COX-2 in a coronary heart disease model, which suggests that PPAR $\gamma, \mathrm{Bax} / \mathrm{Bcl}-2$ and the JAK2/STAT3 signaling pathway were affected by treatment with HSYA. The results of the present study suggest that HSYA may be a potential therapeutic treatment for coronary heart disease in clinical scenarios.

\section{References}

1. Berndt N, Bolman C, Froelicher ES, Mudde A, Candel M, de Vries $\mathrm{H}$ and Lechner L: Effectiveness of a telephone delivered and a face-to-face delivered counseling intervention for smoking cessation in patients with coronary heart disease: A 6-month follow-up. J Behav Med 37: 709-724, 2014

2. Moser DK, McKinley S, Riegel B, Doering LV, Meischke H, Pelter M, Davidson P, Baker H and Dracup K: The impact on anxiety and perceived control of a short one-on-one nursing intervention designed to decrease treatment seeking delay in people with coronary heart disease. Eur J Cardiovasc Nurs 11: 160-167, 2012.

3. Grunwald V, Karakiewicz PI, Bavbek SE, Miller K, Machiels JP, Lee SH, Larkin J, Bono P, Rha SY, Castellano D, et al: An international expanded-access programme of everolimus: Addressing safety and efficacy in patients with metastatic renal cell carcinoma who progress after initial vascular endothelial growth factor receptor-tyrosine kinase inhibitor therapy. Eur J Cancer 48: 324-332, 2012.

4. Derosa G, Bonaventura A, Bianchi L, Romano D, D'Angelo A Fogari E and Maffioli P: A randomized, placebo-controlled study on the effects of a nutraceutical combination of red yeast rice, silybum marianum and octasonol on lipid profile, endothelial and inflammatory parameters. J Biol Regul Homeost Agents 28: 317-324, 2014. 
5. Kuravi SJ, McGettrick HM, Satchell SC, Saleem MA, Harper L, Williams JM, Rainger GE and Savage CO: Podocytes regulate neutrophil recruitment by glomerular endothelial cells via IL-6-mediated crosstalk. J Immunol 193 234-243, 2014

6. Chen G, Qiu H, Ke S, Hu S, Yu S and Zou S: The fibroblast growth factor receptor 2-mediated extracellular signal-regulated kinase $1 / 2$ signaling pathway plays is important in regulating excision repair cross-complementary gene 1 expression in hepatocellular carcinoma. Biomed Rep 1: 604-608, 2013.

7. Haga S, Tsuchiya H,Hirai T, Hamano T, Mimori A and Ishizaka Y: A novel ACE2 activator reduces monocrotaline-induced pulmonary hypertension by suppressing the JAK/STAT and TGF- $\beta$ cascades with restored caveolin-1 expression. Exp Lung Res 41: $21-31,2015$

8. Han JK, Kim HL, Jeon KH, Choi YE, Lee HS, Kwon YW, Jang JJ, Cho HJ, Kang HJ, Oh BH, et al: Peroxisome proliferator-activated receptor- $\delta$ activates endothelial progenitor cells to induce angio-myogenesis through matrix metallo-proteinase-9-mediated insulin-like growth factor-1 paracrine networks. Eur Heart J 34: $1755-1765,2013$

9. Ellis HP and Kurian KM: Biological rationale for the use of PPAR $\gamma$ agonists in glioblastoma. Front Oncol 4: 52, 2014

10. Aydoğan HY, Küçükhüseyin O, Tekeli A and Isbir T: Associations of receptor for advanced glycation end products-374 T/A and Gly82 Ser and peroxisome proliferator-activated receptor gamma Pro12Ala polymorphisms in Turkish coronary artery disease patients. Genet Test Mol Biomarkers 16: 134-137, 2012.

11. Huang TH and Roufogalis BD: Healing the diabetic heart: Modulation of cardiometabolic syndrome through peroxisome proliferator activated receptors (PPARs). Curr Mol Pharmacol 5: 241-247, 2012

12. Zhang Z, Wu Z, Zhu X, Hui X, Pan J and Xu Y: Hydroxy-safflor yellow A inhibits neuroinflammation mediated by $\mathrm{A} \beta(1)(-)(4)(2)$ in BV-2 cells. Neurosci Lett 562: 39-44, 2014.

13. Zhou MX, Fu JH, Zhang Q and Wang JQ: Effect of hydroxy safflower yellow A on myocardial apoptosis after acute myocardial infarction in rats. Genet Mol Res 14: 3133-3141, 2015.

14. Livak KJ and Schmittgen TD: Analysis of relative gene expression data using real-time quantitative PCR and the 2(-Delta Delta C(T)) method. Methods 25: 402-408, 2001.

15. Kaysen GA, Levin NW, Mitch WE, Chapman AL, Kubala L and Eiserich JP: Evidence that C-reactive protein or IL-6 are not surrogates for all inflammatory cardiovascular risk factors in hemodialysis patients. Blood Purif 24: 508-516, 2006.

16. Dusting GJ: Nitric oxide in coronary artery disease: Roles in atherosclerosis, myocardial reperfusion and heart failure. EXS 76: 33-55, 1996.
17. Baptista J, Teles RC, da Silva PC, Pereira H, Marques L, Santos R, Carvalho H, Martins D, Farto e Abreu P, Araújo J, et al: Five-year clinical results of coronary angioplasty with drug-eluting stents. National initiative in strategic innovation, iNOS. Rev Port Cardiol 29: 243-251, 2010 (In English, Portuguese).

18. Meng QH, Irvine S, Tagalakis AD, McAnulty RJ, McEwan JR and Hart SL: Inhibition of neointimal hyperplasia in a rabbit vein graft model following non-viral transfection with human iNOS cDNA. Gene Ther 20: 979-986, 2013.

19. Wisel S, Khan M, Kuppusamy ML, Mohan IK, Chacko SM, Rivera BK, Sun BC, Hideg K and Kuppusamy P: Pharmacological preconditioning of mesenchymal stem cells with trimetazidine (1-[2,3,4-trimethoxybenzyl]piperazine) protects hypoxic cells against oxidative stress and enhances recovery of myocardial function in infarcted heart through $\mathrm{Bcl}-2$ expression. J Pharmacol Exp Ther 329: 543-550, 2009.

20. Wang Y, Zhang H, Chai F, Liu X and Berk M: The effects of escitalopram on myocardial apoptosis and the expression of Bax and $\mathrm{Bcl}-2$ during myocardial ischemia/reperfusion in a model of rats with depression. BMC Psychiatry 14: 349, 2014.

21. Roxburgh CS and McMillan DC: Therapeutics targeting innate immune/inflammatory responses through the interleukin-6/JAK/STAT signal transduction pathway in patients with cancer. Transl Res 167: 61-66, 2016.

22. Wen SH, Li Y, Li C, Xia ZQ, Liu WF, Zhang XY, Lei WL, Huang WQ and Liu KX: Ischemic postconditioning during reperfusion attenuates intestinal injury and mucosal cell apoptosis by inhibiting JAK/STAT signaling activation. Shock 38: 411-419, 2012.

23. Mo ZC, Xiao J, Liu XH, Hu YW, Li XX, Yi GH, Wang Z, Tang YL, Liao DF and Tang CK: AOPPs inhibits cholesterol efflux by down-regulating ABCA1 expression in a JAK/STAT signaling pathway-dependent manner. J Atheroscler Thromb 18: 796-807, 2011.

24. Lu Y,Zhou J, Xu C, Lin H, Xiao J, Wang Z and Yang B: JAK/STAT and PI3K/AKT pathways form a mutual transactivation loop and afford resistance to oxidative stress-induced apoptosis in cardiomyocytes. Cell Physiol Biochem 21: 305-314, 2008.

25. Negoro S, Kunisada K, Tone E, Funamoto M, Oh H, Kishimoto T and Yamauchi-Takihara K: Activation of JAK/STAT pathway transduces cytoprotective signal in rat acute myocardial infarction. Cardiovasc Res 47: 797-805, 2000. 